Check for updates

Cite this: RSC Adv., 2019, 9, 22677

Received 9th April 2019

Accepted 1st July 2019

DOI: $10.1039 / \mathrm{c} 9 \mathrm{ra02665g}$

rsc.li/rsc-advances

\section{Application of natural deep eutectic solvents to extract ferulic acid from Ligusticum chuanxiong Hort with microwave assistance}

\author{
Yilin Xie, $\uparrow$ Herui Liu, $\uparrow$ Li Lin, Maojun Zhao, (D)* Li Zhang, Yunsong Zhang \\ and Yichao Wu $\mathrm{WD}$
}

\begin{abstract}
In this study, a method using natural deep eutectic solvents (NADES) combined with microwave-assistance extraction (MAE) was researched for the first time to establish an environmentally-friendly method for extracting ferulic acid from Ligusticum chuanxiong Hort. 20 kinds of NADES were initially screened, then response surface methodology was performed to optimize the NADES-MAE extraction of ferulic acid in $L$. $c$ on the basis of the results of single-factor experiments. The results demonstrated that NADES could provide better extraction yields of ferulic acid than conventional solvents, and the combination of choline chloride and 1,2-propanediol was the most effective. The optimal conditions were an extraction time of $20 \mathrm{~min}$, an extraction temperature of $68^{\circ} \mathrm{C}$, and a solvent-to-solid ratio of $30: 1 \mathrm{~mL} \mathrm{~g}^{-1}$. Under these conditions, the extraction yield of ferulic acid with NADES-MAE $\left(2.32 \mathrm{mg} \mathrm{g}^{-1}\right)$ was higher than that using traditional extraction methods. This research demonstrates that this approach, which adopts NADES as a green solvent and MAE as an assistant extraction technique, could be an excellent choice to design an environmentally-friendly method for extracting phenolic compounds in various materials.
\end{abstract}

\section{Introduction}

Chuanxiong rhizoma, the dried root of umbelliferous Ligusticum chuanxiong Hort, is a common traditional herbal medicine widely used in Asia. ${ }^{1}$ L. chuanxiong as an important drug, which is commonly applied to promote blood circulation in Chinese medicine clinical trials, can be used alone as well as with other traditional herbs. It is a component of many traditional Chinese patent medicines ${ }^{2,3}$ which are widely used in treating headaches and cardiovascular diseases such as angina and ischemic stroke. It is also a major ingredient in soups for regular consumption to promote good body health and has a positive effect in guarding against dizziness. ${ }^{4}$ In addition, the tender stem and leaves of $L$. chuanxiong can be cooked in salad or fried cuisines. ${ }^{1}$ L. chuanxiong contains a wide range of bioactive components including tetramethylpyrazine, ferulic acid and phthalide compounds. ${ }^{5}$ Among them, ferulic acid is the main liposoluble ingredient in L. chuanxiong, and has been recommended as the biomarker for L. chuanxiong in the Pharmacopoeia of the People's Republic of China. ${ }^{6,7}$ Since ferulic acid has many pharmaceutical activities, such as its antioxidant activity, its ability to treat cardiovascular disease, its antibacterial activity and its anti-cancer activity, it is widely investigated. In addition, ferulic acid has been approved as an antioxidant and

College of Science, Sichuan Agricultural University, Ya'an, Sichuan 625014, China. E-mail: Zhaomj2019@163.com

$\dagger$ These authors contributed equally to the paper. food preservative in Japan. ${ }^{8,9}$ Sodium ferulate, a sort of ferulic acid salt, has been used in China to treat cardiovascular and cerebrovascular diseases. ${ }^{10}$ Therefore, it is of great importance to develop a green and efficient method to extract ferulic acid from $L$. chuanxiong.

At present, the classic approaches of extracting ferulic acid were heating combined with organic solvents ${ }^{11-16}$ and supercritical extraction, ${ }^{\mathbf{1 7}}$ which have many problems such as their low efficiency, ${ }^{11-14}$ long times, ${ }^{11-16}$ high temperature ${ }^{11,12,15,16}$ and high cost of the solvents. ${ }^{17}$ There are main two directions to improve those problems. One aspect is to apply external forces like microwaves ${ }^{18}$ ultrasonics $^{19}$ or ultrahigh voltage waves ${ }^{20}$ to the mixture of materials and solvents for the purpose of reducing the time taken, lowering the extraction temperature and improving the extraction efficiency. MAE is an extraction technique that utilizes microwaves as the external force to extract components. Compared with other extraction techniques (such as reflux and supercritical fluid extraction), it is a faster and greener method. MAE can accomplish the processes of heat transfer and mass transfer of the target components from sample to solvent at the same time, thus greatly reducing the consumption of time and organic solvents. ${ }^{21,22}$ Additionally, MAE has been wildly used in extracting bioactive components, including phenol components in diverse medicine materials ${ }^{23-26}$ and ferulic acid and phthalides in L. chuanxiong. ${ }^{\mathbf{1 8 2 7}}$ Another research direction is to find green and efficient solvents that can replace the traditional harmful ones (such as methanol, ethanol, ethyl acetate and so 
on). In recent several years NADES, which were regarded as a green substitute for traditional solvents, have attracted much interest. ${ }^{28,29}$ NADES are usually mixtures that consist of two or more kinds of naturally occurring and biodegradable components (such as glucose, an organic acid, polyalcohol and choline chloride $)^{30,31}$ and can be prepared by green and fast methods such as heating ${ }^{32,33}$ and microwave-assisted methods. ${ }^{34}$ The physicochemical properties of NADES are similar to those of ionic liquids. But compared with ionic liquids, NADES provide many advantages, such as readily available components, simple preparation, low toxicity and tunability. ${ }^{35,36}$ Some studies have reported the applications of NADES in the extraction of phenolic acids and phenolic compounds, ${ }^{31,36-38}$ which have had good results. What's more, MAE combined with various NADES has been used to extract phenolic compounds. ${ }^{39-41}$ However, there is no study on the application of MAE combined with NADES for extracting ferulic acid from $L$. chuanxiong.

The purpose of this study was to build up an efficient and truly environmentally-friendly method for extracting ferulic acid from L. chuanxiong with the help of NADES and MAE. At first, preliminary screening for the optimal NADES via the maximum yield of ferulic acid was performed. And the effect of important parameters, namely the molar ratio of NADES, water content of NADES, extraction time, extraction temperature and solvent-tosolid ratio, on the extraction yield were explored. Then the optimal extraction conditions of NADES-MAE were determined by response surface methodology (RSM) successfully. Finally, the properties of the optimal method were evaluated.

\section{Materials and methods}

\subsection{Chemicals and materials}

L. chuanxiong was purchased from a local Chinese medicine herb market (Chengdu, China), dried at $60{ }^{\circ} \mathrm{C}$ until it reached the stable weight, crushed and pressed through a 40-mesh sieve, then kept at $-20{ }^{\circ} \mathrm{C}$ prior to the experimental analysis.

A ferulic acid standard was obtained from Chengdu Must Biological Technology Co., Ltd (Chengdu, China). Choline chloride, ethylene glycol, 1,2-propanediol, glycerol, glucose, sucrose, maltose, malic acid, citric acid, lactic acid, oxalic acid, ethanol, methanol and other chemicals were purchased from Chengdu Kelong chemical reagent factory (Sichuan, China). Deionized water (electrical resistivity $\approx 18.2 \mathrm{M} \Omega \mathrm{cm}$ ) used in the experiments was purified by a water-purifying system of Sichuan Water Technology Development Co., Ltd.

\subsection{Preparation of NADES}

According to previous research, ${ }^{32,33}$ NADES can be easily synthesized using a heating method. As shown in Table 1, twenty NADES were prepared at $60{ }^{\circ} \mathrm{C}$ by mixing choline chloride, 1,2-propanediol, glucose and other components that were weighed accurately according to the proper molar ratio with constant stirring until a homogeneous liquid formed.

\subsection{Extraction of ferulic acid in L. chuanxiong}

Samples of $0.5 \mathrm{~g}$ of powdered material were extracted with $10 \mathrm{~mL}$ of different solvents $\left(\mathrm{H}_{2} \mathrm{O}, \mathrm{MeOH}, \mathrm{EtOH}, 70 \% \mathrm{MeOH}\right.$, $70 \%$ EtOH and 20 sorts of NADES) by microwave-assisted extraction (MAE). The mixture was processed using a microwave (MAS-1, $1360 \mathrm{~W}$, Shanghai, China) at $60{ }^{\circ} \mathrm{C}$ for $15 \mathrm{~min}$, and then centrifuged at $10000 \mathrm{rmp}$ for $5 \mathrm{~min}$. The supernatant liquid was diluted with methanol for HPLC analysis. These experiments were repeated in triplicate.

\subsection{Quantitative analysis of HPLC}

The quantitative analysis of HPLC was performed on an Agilent 1260 series liquid chromatography system and the separation

Table 1 Different systems of natural deep eutectic solvents ${ }^{a}$

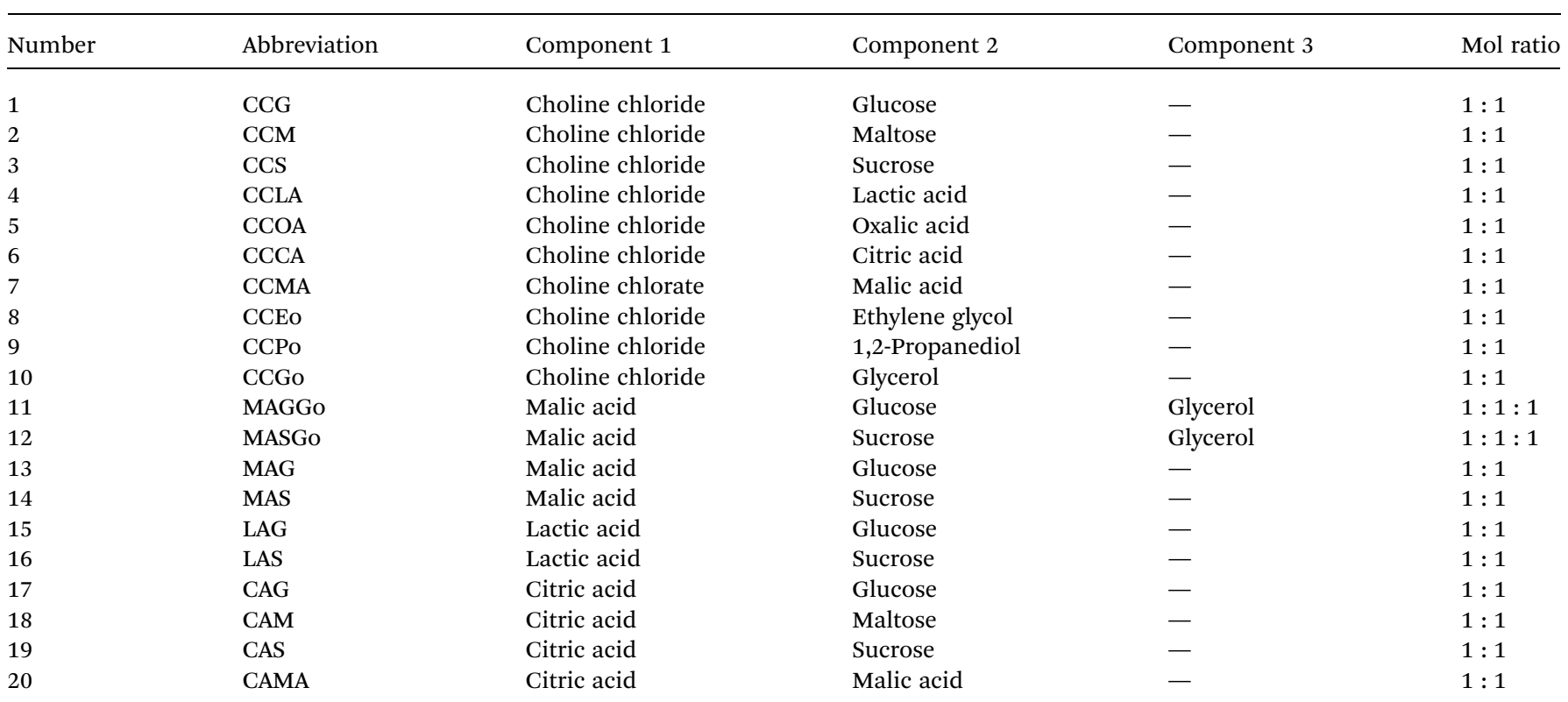

${ }^{a}$ _: not added. 
was carried out on an InterSustain C18 column $(50 \mathrm{~mm} \times 4.6$ $\mathrm{mm}, 5 \mu \mathrm{m}$ ). The mobile phases were: (A) $0.1 \%(\mathrm{v} / \mathrm{v})$ aqueous phosphoric acid and (B) methanol. The gradient conditions were performed as follows: $0-5 \mathrm{~min}, 30 \% \mathrm{~B}$; 5-12 $\mathrm{min}, 30-43 \%$ B; 12-17 min, 43\% B; 17-19 min, 43-79\% B; 19-30 min, 79\% B. The column was operated at $35{ }^{\circ} \mathrm{C}$ with the mobile phase at a constant flow rate of $1 \mathrm{~mL} \mathrm{~min}^{-1}$, and the detection wavelength was set at $320 \mathrm{~nm}$. Each run was followed by an equilibration period of $5 \mathrm{~min}$. All the samples were filtered with 0.22 $\mu \mathrm{m}$ Whatman (Piscataway, NJ, USA) filter membranes. The injection volume was $10 \mu \mathrm{L}$. The chromatographic peaks of the target component were identified by comparing the retention time with the standard compound. Quantitative analysis of the target component was confirmed by applying the method of standard curves. HPLC chromatograms of the standard compound and sample are presented in Fig. 1.

\subsection{Optimization of extraction parameters}

The parameters of ferulic acid extracted by NADES were optimized. The parameters included the molar ratio of the components in the NADES $(1: 0.2,1: 0.25,1: 0.33,1: 0.5,1: 1$, $1: 2,1: 3,1: 4$ and $1: 5)$, the water content in the NADES $(0 \%$, $10 \%, 20 \%, 30 \%, 40 \%, 50 \%, 60 \%$ and $70 \%)$, the extraction time $(2,5,10,15,20,25$ and $30 \mathrm{~min})$, the extraction temperature $(40$, $50,60,70$ and $\left.80{ }^{\circ} \mathrm{C}\right)$, and the solvent-to-solid ratio $(5: 1,10: 1$, $15: 1,20: 1,25: 1,30: 1$ and $35: 1 \mathrm{~mL} \mathrm{~g}^{-1}$ ).

\subsection{Experimental design and statistical analysis}

In this study, the Box-Behnken design (BBD) combined with RSM was used to research the optimal NADES-MAE conditions. On the basis of the single factor experiment results, three factors as independent variables, namely the extraction time (A), extraction temperature (B) and solvent-to-solid ratio (C), were investigated at three levels (Table 2).

The experimental data were analyzed to fit the following second-order polynomial model:

$$
Y=\beta_{0}+\sum_{i=1}^{3} \beta_{i} X_{i}+\sum_{i=1}^{3} \beta_{i i} X_{i}^{2}+\sum_{i=1}^{2} \sum_{j=i+1}^{3} \beta_{i j} X_{i} X_{j}
$$

Table 2 Independent factors and their levels used in MAE

\begin{tabular}{lllll}
\hline & \multicolumn{5}{l}{ Levels } \\
\cline { 4 - 5 } Independent factor & Symbol & -1 & 0 & 1 \\
\hline Extraction time (min) & $\mathrm{A}$ & 10 & 15 & 20 \\
Extraction temperature $\left({ }^{\circ} \mathrm{C}\right)$ & $\mathrm{B}$ & 50 & 60 & 70 \\
Solvent-to-solid ratio $\left(\mathrm{mL} \mathrm{g}^{-1}\right)$ & $\mathrm{C}$ & $20: 1$ & $25: 1$ & $30: 1$ \\
\hline
\end{tabular}

where $Y$ is the response variable; $\beta_{0}$ is a constant; $\beta_{i}, \beta_{i i}$ and $\beta_{i j}$ are the liner, quadratic and second-order terms of the model, respectively; and $X_{i}$ and $X_{j}$ are independent variables.

All the statistical analysis was carried out with the help of Design Expert v 8.0.6. The statistical significance of the model obtained by BBD was inspected by analysis of variance (ANOVA). Additionally, according to the $P$ value the interactions of each coefficient were evaluated.

\section{Results and discussion}

\subsection{Screening of NADES composition}

In this study, 20 different types of NADES were prepared by 11 kinds of primary metabolites, and their extraction yields of ferulic acid from $L$. chuanxiong were tested. However, the obvious disadvantage of NADES is their high viscosity, which could lower the mass transport efficiency. ${ }^{21,32}$ Therefore, the extraction temperature and time were $60^{\circ} \mathrm{C}$ and $15 \mathrm{~min}$, and the extraction solvent used was NADES with the addition of $30 \%(\mathrm{w} /$ w) water. Five conventional solvents, namely $\mathrm{H}_{2} \mathrm{O}, \mathrm{MeOH}, \mathrm{EtOH}$, $70 \% \mathrm{MeOH}$ and $70 \% \mathrm{EtOH}$ were used as contrastive solvents.

As shown in Fig. 2a, the extraction yields of NADES were higher than those of conventional solvents, indicating that NADES displayed an obvious advantage for the extraction of ferulic acid. Among the 20 kinds of NADES, the extraction yield of the target compound with the different types of NADES differed. The polyalcohol-based NADES had equivalent or even higher extraction yields compared with those of the saccharidebased and organic acid-based NADES. The extraction yields of organic acid-saccharide-glycerol-based NADES (Mac-Glu-Gly

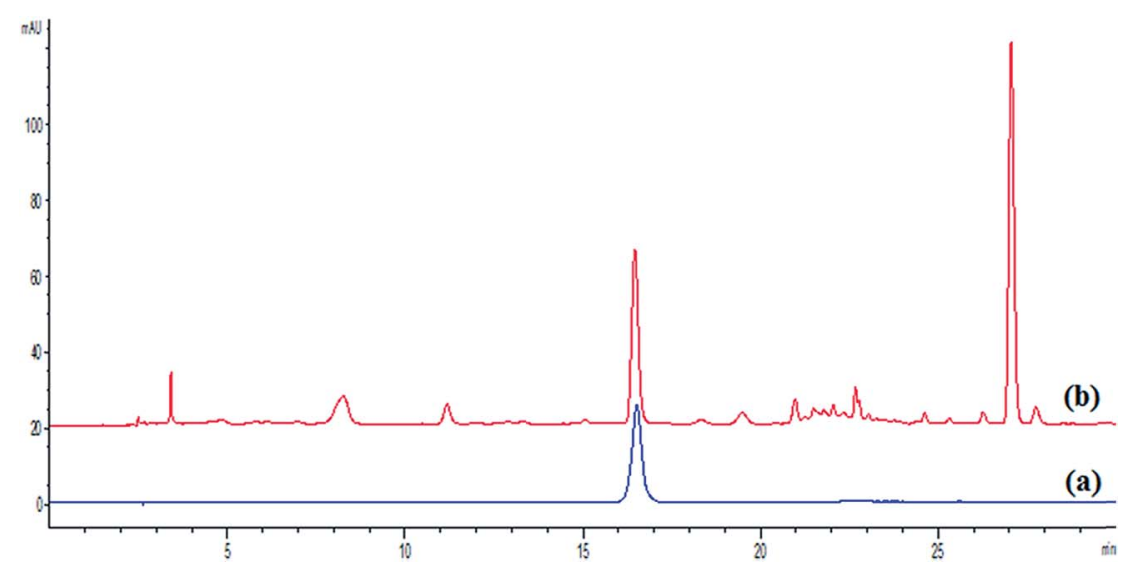

Fig. 1 HPLC chromatograms of ferulic acid at $320 \mathrm{~nm}$ : (a) reference compounds and (b) sample. 


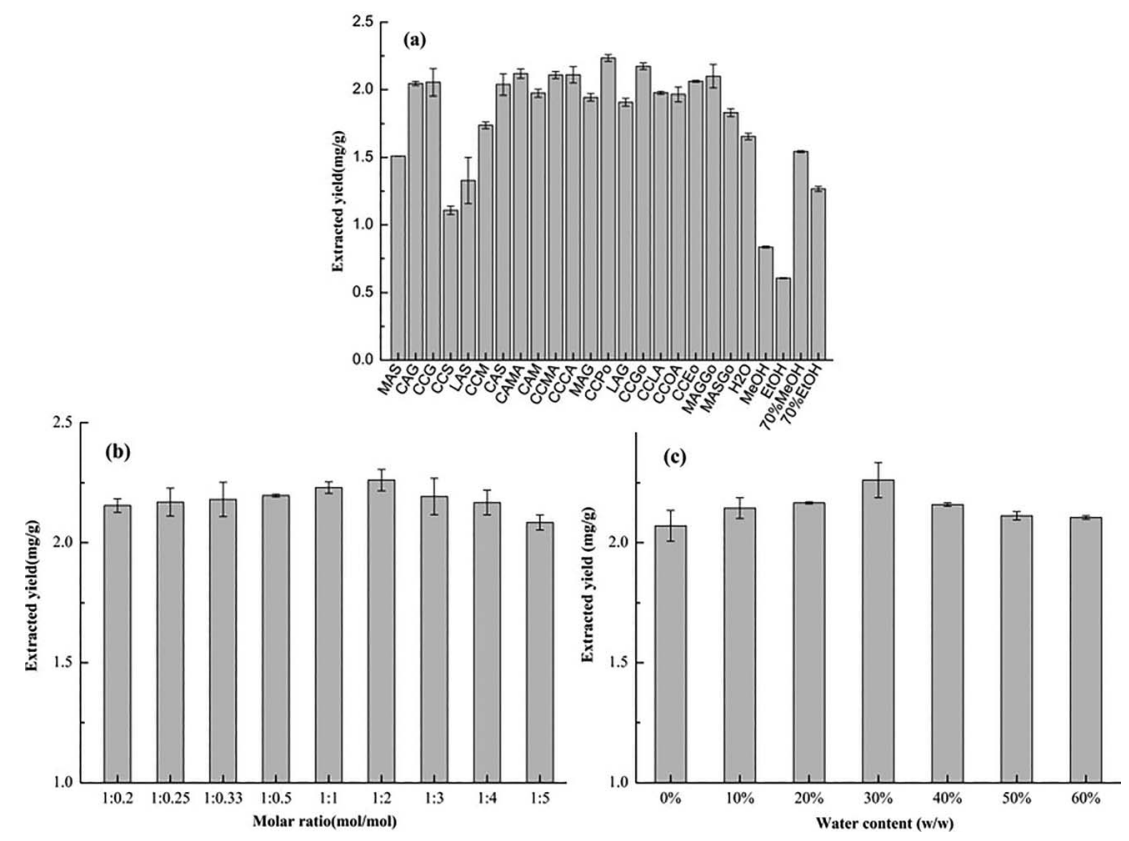

Fig. 2 Extraction yields with different solvents (a), molar ratios (b) and water content (c) for ferulic acid.

and Mac-Su-Gly) were higher than those of the organic acidsaccharide-based ones (Mac-Glu and Mac-Su), which indicated that the existence of polyalcohol benefits the increase of extraction yield. Additionally, the extraction yield of lactic-acidbased NADES were lower than that of the polyalcohol-based NADES. The extraction yield seems to be closely related to the properties of the solvents and the possible interaction between solutes and solvents. ${ }^{42}$ Therefore, the reasons for those results were not only the effect of solvent viscosity, but more importantly, the effect of hydrogen bonds formed between NADES and the target components. Meanwhile, among the NADES containing polyalcohol, CCPo exhibited the highest extraction yield. So CCPo was chosen as the final component of NADES.

Fig. $2 \mathrm{~b}$ reveals the influence of the different NADES component ratios on the extraction yield. The results show that the ratio change of choline chloride/1,2-propanediol could partly influence the extraction yield of ferulic acid from $L$. chuanxiong. Increasing the choline chloride/1,2-propanediol ratio from $1: 0.2$ to $1: 2$ enhanced the extraction yield of ferulic acid. This may be because the solvent viscosity and the number of hydrogen bonds changed, thus contributing to the breakage of the plant cell wall and the dissolution of target components. However, a further increase in the ratio negatively affected the extraction yield. Therefore, the combination of choline chloride/1,2-propanediol with a molar ratio of $1: 2$ (mol $\mathrm{mol}^{-1}$ ) was chosen as the most promising NADES for further research.

As well as the molar ratio of solvent components, the different water content of the NADES could also affect the viscosity of the solvents, leading to differences in extraction yield. ${ }^{43}$ Therefore, the extraction yields of NADES with water content ranging from $0 \%$ to $60 \%$ were researched. Fig. $2 \mathrm{c}$ shows that the extraction yield of ferulic acid changed with the changing water content. For instance, the extraction process was improved as the water content in NADES increased from $0 \%$ to $30 \%$. This is probably because of the reduction in solvent viscosity caused by adding water which would help with the process of mass transfer from materials to solvents. ${ }^{44}$ Nevertheless, as the water content increased further $(30-60 \%)$, the extraction yield declined. These phenomena might be associated with the change of interaction between the target component and NADES caused by the high content of water. ${ }^{45}$ Therefore, a water content of $30 \%$ was determined to be the best choice.

\subsection{Optimisation of NADES-MAE conditions}

According to the results of single-factor experiments, seventeen experiments were designed and accomplished using BBD of RSM in order to fix the optimal conditions of NADES-MAE. The results are listed as Table 3.

Polynomial regression analysis was performed to analyze the experiment data using Design Expert 8.0.6 software. The obtained polynomial model was as follows:

$$
\begin{aligned}
Y= & -1.74+0.0539 X_{1}+0.105 X_{2}-0.005 X_{3} \\
& -0.0006 X_{2}-0.0008 X_{1} X_{3}+0.0007 X_{2} X_{3} \\
& +0.00042 X_{1}^{2}-0.000845 X_{2}{ }^{2}-0.00038 X_{3}^{2}
\end{aligned}
$$

where $Y$ is the extraction yield of ferulic acid and A, B and C are variables that represent extraction time, extraction temperature and the solvent-to-solid ratio, respectively.

ANOVA was employed in analyzing the polynomial regression model in order to estimate the optimized conditions of NADES-MAE and the relationships between response value and variables. The results are listed in Table 4 . A model $F$-value of 56.52 and $P$-value of $<0.0001$ indicated that the model was 
Table 3 Box-Behnken design of independent variables and obtained data

\begin{tabular}{|c|c|c|c|c|}
\hline \multirow[b]{2}{*}{ Run } & \multicolumn{3}{|c|}{ Factor } & \multirow{2}{*}{$\begin{array}{l}\text { Extraction yield } \\
\left(\mathrm{mg} \mathrm{g}^{-1}\right)\end{array}$} \\
\hline & $\mathrm{A}$ & B & $\mathrm{C}$ & \\
\hline 1 & 10 & 50 & 25 & 2.00 \\
\hline 2 & 20 & 60 & 30 & 2.33 \\
\hline 3 & 10 & 60 & 20 & 2.18 \\
\hline 4 & 20 & 50 & 25 & 2.18 \\
\hline 5 & 10 & 70 & 25 & 2.28 \\
\hline 6 & 15 & 60 & 25 & 2.28 \\
\hline 7 & 15 & 70 & 20 & 2.25 \\
\hline 8 & 15 & 60 & 25 & 2.28 \\
\hline 9 & 15 & 70 & 30 & 2.38 \\
\hline 10 & 15 & 60 & 25 & 2.25 \\
\hline 11 & 15 & 60 & 25 & 2.29 \\
\hline 12 & 20 & 70 & 25 & 2.34 \\
\hline 13 & 15 & 50 & 30 & 2.04 \\
\hline 14 & 15 & 60 & 25 & 2.27 \\
\hline 15 & 15 & 50 & 20 & 2.05 \\
\hline 16 & 10 & 60 & 30 & 2.28 \\
\hline 17 & 20 & 60 & 20 & 2.31 \\
\hline
\end{tabular}

significant. A $P$-value of lack of fit item $0.2020(>0.05)$ indicated that the model was of great veracity and stability. The correlation coefficient $R^{2}(0.9730)$ and adj. $R^{2}(0.9390)$ was close to 1 , indicating the good accuracy and fitness of the model. The C.V.\% was 0.87 , demonstrating that the model was feasible to predict the extraction yield of ferulic acid. In addition, as shown in Table 4 , the liner terms of $A, B$ and $C$ and quadratic term of $B^{2}$ were extraordinarily significant $(P<0.01)$ and the terms of $\mathrm{AB}$ and $\mathrm{BC}$ were significant $(P<0.05)$. The order of factors that affected extraction yield was: extraction temperature $>$ extraction time $>$ solvent-to-solid ratio.

Three-dimensional (3D) response surface plots, as an important part of the regression equation, vividly showed the

Table 4 ANOVA of quadratic model for the extraction yields of ferulic acid

\begin{tabular}{llllll}
\hline Source & Sum of squares & DF & Mean square & $F$ value & $p$-Value \\
\hline Model & 0.19 & 9 & 0.021 & 56.52 & $<0.0001$ \\
$\mathrm{~A}$ & 0.022 & 1 & 0.022 & 58.91 & 0.0001 \\
$\mathrm{~B}$ & 0.12 & 1 & 0.12 & 320.74 & $<0.0001$ \\
$\mathrm{C}$ & $7.200 \times 10^{-3}$ & 1 & $7.200 \times 10^{-3}$ & 19.24 & 0.0032 \\
$\mathrm{AB}$ & $3.600 \times 10^{-3}$ & 1 & $3.600 \times 10^{-3}$ & 9.62 & 0.0173 \\
$\mathrm{AC}$ & $1.600 \times 10^{-3}$ & 1 & $1.600 \times 10^{-3}$ & 4.27 & 0.0775 \\
$\mathrm{BC}$ & $4.900 \times 10^{-3}$ & 1 & $4.900 \times 10^{-3}$ & 13.09 & 0.0085 \\
$\mathrm{~A}^{2}$ & $4.642 \times 10^{-4}$ & 1 & $4.642 \times 10^{-3}$ & 1.24 & 0.3022 \\
$\mathrm{~B}^{2}$ & 0.030 & 1 & 0.030 & 80.32 & $<0.0001$ \\
$\mathrm{C}^{2}$ & $3.800 \times 10^{-4}$ & 1 & $3.800 \times 10^{-3}$ & 1.02 & 0.3472 \\
Residual & $2.620 \times 10^{-3}$ & 7 & $3.743 \times 10^{-3}$ & & \\
Lack of fit & $1.700 \times 10^{-3}$ & 3 & $5.667 \times 10^{-3}$ & 2.46 & 0.2020 \\
Pure error & $9.200 \times 10^{-4}$ & 4 & $2.300 \times 10^{-3}$ & & \\
Cor total & 0.19 & 16 & & & \\
Std. dev. & 0.019 & & $R^{2}$ & 0.9864 & \\
Mean & 2.23 & & Adj $R^{2}$ & 0.9690 & \\
C.V. \% & 0.87 & & Pred $R^{2}$ & 0.8516 & \\
PRESS & 0.029 & & Adeq precision & 25.104 & \\
& & & & &
\end{tabular}

interactions between two parameters. For the convenience of description, with one factor fixed, the other two factors were changed within defined limits in the present study. Fig. 3 illustrates that the extraction yield increased with increasing time. And a high extraction yield can be obtained at $20 \mathrm{~min}$. This may be the result of the microwave's unique heating style that can complete the processes of heat and mass transfer at the same time. ${ }^{46}$ When the temperature rises, the extraction yield is improved as well. This may be because the high temperature could reduce the physical and chemical interactions between the target components and matrices and facilitate the NADES's penetration, thereby improving the mass transfer of the target component from the interior plant matrices to the NADES. ${ }^{47}$ However, if the temperature was over $68^{\circ} \mathrm{C}$, the extraction yield declined slightly. On increasing the solvent-to-solid ratio, the extraction yield increased, but not significantly, especially at a long extraction time and a middling temperature. All the consequences indicated that high temperatures, high solventto-solid ratios and long extraction times are not suitable for the extraction of ferulic acid.

\subsection{Model validation and method comparison}

Through the BBD experiment, the optimal conditions were determined as follows: an extraction time of $20 \mathrm{~min}$, an extraction temperature of $67.54{ }^{\circ} \mathrm{C}$, and a solvent-to-solid ratio of $30: 1 \mathrm{~mL} \mathrm{~g}^{-1}$. For convenience, experimental extractions were conducted using the following values: an extraction time of $20 \mathrm{~min}$, an extraction temperature of $68{ }^{\circ} \mathrm{C}$, and a solvent-tosolid ratio of $30: 1 \mathrm{~mL} \mathrm{~g}^{-1}$. Under these optimal extraction conditions, the experimental value for ferulic acid was determined to be $2.32 \mathrm{mg} \mathrm{g}^{-1}$ (0.51\% RSD), which resided within the $95 \%$ prediction interval of $2.39 \mathrm{mg} \mathrm{g}^{-1}$. These data implied that the quadratic model which was applied to predict the extraction yield was reliable. In addition, compared with the methods of heating-73\% $\mathrm{EtOH}^{48}\left(1.52 \mathrm{mg} \mathrm{g^{-1 }}\right)$ and MAE-40\% $\mathrm{EtOH}^{18}$ $\left(0.77 \mathrm{mg} \mathrm{g}^{-1}\right)$, the optimized NADES-MAE method offered a higher extraction yield of ferulic acid.

The published methods ${ }^{48}$ of extracting ferulic acid from $L$. chuanxiong with slight modifications were applied to compare them with the optimal method in this study for the sake of evaluating the latter's extraction efficiency (Table 5). Using EtOH as the extraction solvent, the yield of MAE (ref-3) was higher than that of the heating method (ref-1), and there was a similar result using NADES as the solvent, which indicated that the optimized MAE could take less time and energy for efficiently extracting ferulic acid. On the other hand, while extracting by the same methods (heating or MAE), the yield for NADES was higher than that for $70 \%$ EtOH. Similarly, as shown in Fig. 2, the same results appeared in the comparison between NADES and traditional solvents. All these consequences demonstrated that NADES have advantages over the traditional solvents and can efficiently improve the extraction yield. Meanwhile this solvent chosen is safe and green. Therefore, the NADES-MAE method is a promising environmentally-friendly and efficient method for the extraction of active ingredients from plant samples. 

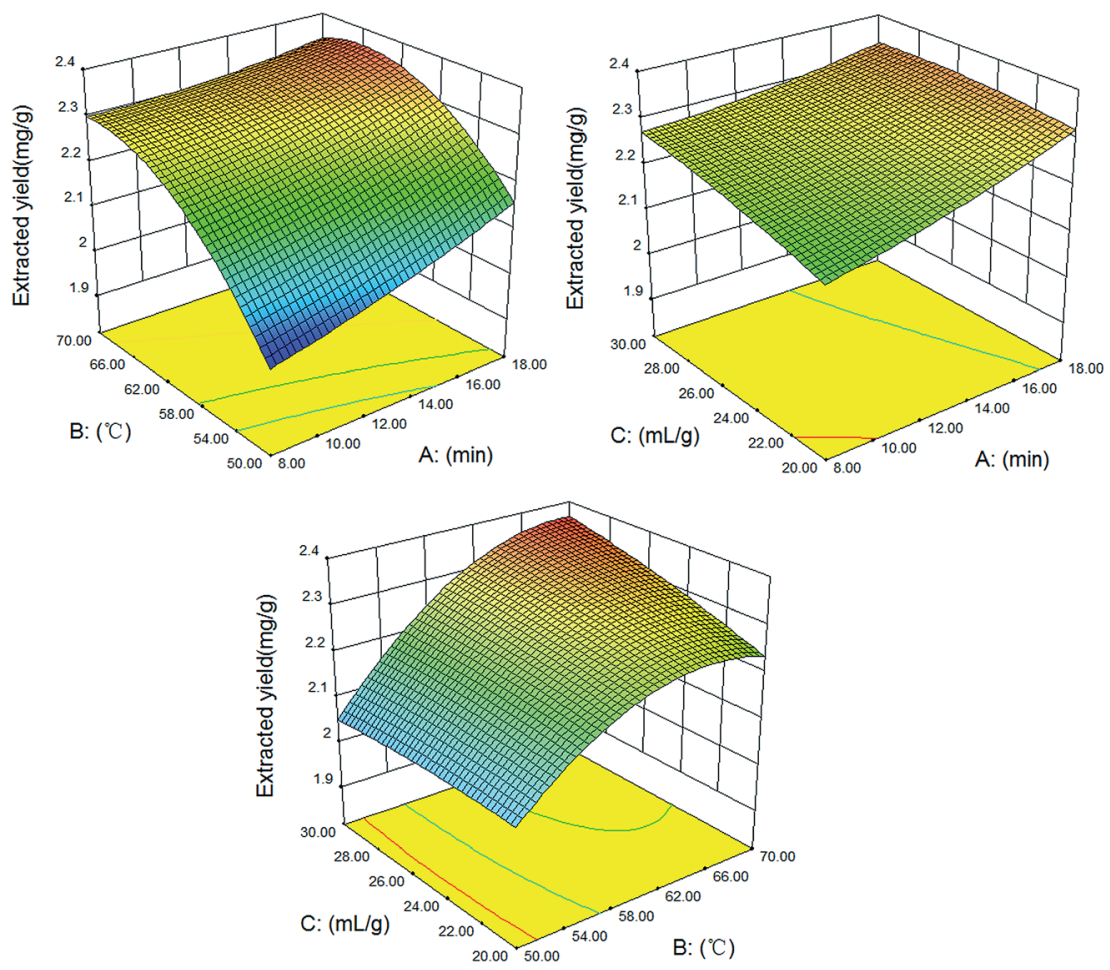

Fig. 3 3D response surface plots of the interactions between three extraction factors (extraction time, extraction temperature and the solventto-solid ratio)

\subsection{Exploration of the industrial application potential}

In this report, MAE was applied as the extraction method because of the advantages of high efficiency and convenience of operation. Other NADES-compatible extraction methods, such as ultrasound assisted extraction (UAE), stirring, heating, and heating + stirring were also investigated (Fig. 4). Among the five extraction methods, the extraction yield of MAE was the highest, followed by heating + stirring and heating extraction, and the yields of stirring and UAE were significantly lower than those of MAE or heating + stirring $(p<0.001)$. These results imply that the methods of heating or heating + stirring can replace the work of MAE when the latter is not available or when the scale of the required extraction is larger than that can be performed in microwave conditions.

Based on the findings above, to explore the application potential of the optimal method in industry, the method was tested to determine whether it could be applied for large-scale $L$. chuanxiong extraction. In general, a large-scale extraction should be accompanied by a large volume of extraction solvent. In this report, the NADES was prepared by the fast and convenient heating method and diluted to a water content of $30 \%$ for the extraction solvent. The extraction solvent volume was expanded 50-fold to $750 \mathrm{~mL}$ and for technical ease the heating + stirring method was applied for the large-scale extraction. The extraction yield of ferulic acid measured at $20 \mathrm{~min}$ was $1.98 \mathrm{mg}$ $\mathrm{g}^{-1}$, which was $85 \%$ of that acquired by NADES-MAE $(2.32 \mathrm{mg}$ $\mathrm{g}^{-1}$ ), and increased in a time-dependent manner. After $70 \mathrm{~min}$ extraction, the extraction yield of the large-scale process was not markedly different from the small-scale one, while the former reached $99 \%$ of the latter after 90 min (Fig. 5). All the results above revealed that the optimal conditions of small-scale extraction cannot be transferred directly to large-scale ones. Despite this, the NADES still performed well in the large-scale extraction, the yield of which is comparable with that of the lab-scale extraction under the properly modified conditions, indicating that NADES has the potential of being applied in industrial production. In addition, some studies concerning the

Table 5 Comparison of the optimal method with reference methods

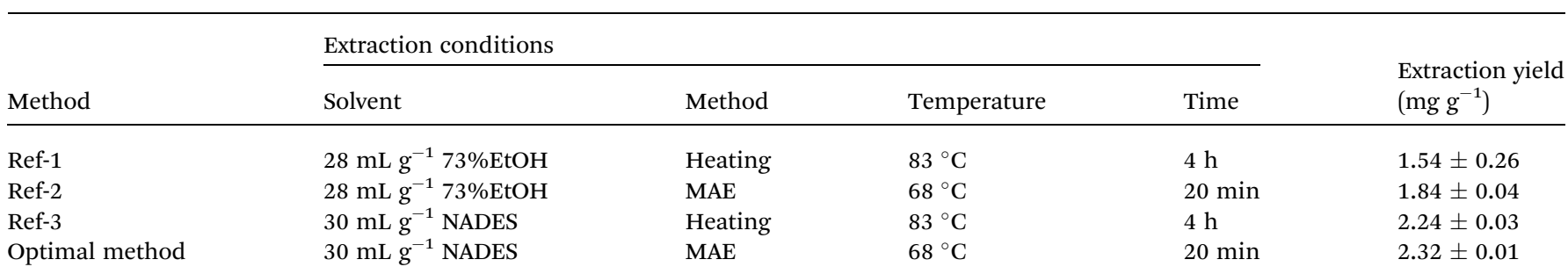




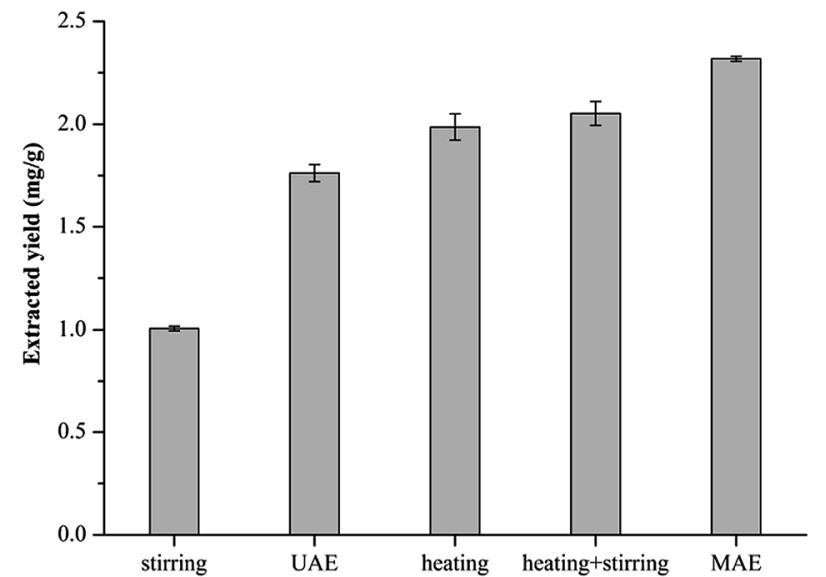

Fig. 4 Ferulic acid yields for the five different extraction methods using CCPO.

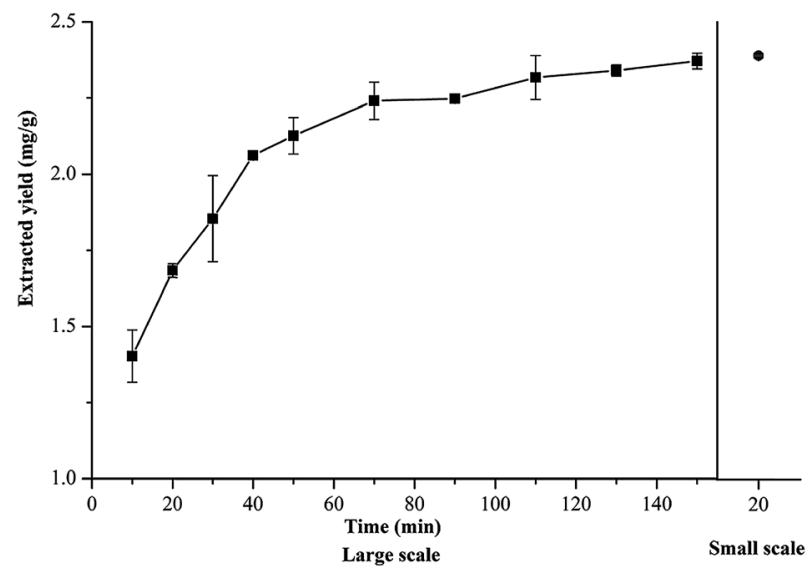

Fig. 5 Ferulic acid extraction yield acquired in a 50-fold scaled-up experiment (left) and a small-scale experiment (right).

cost of applying NADES reported the practicability of applying NADES in industry production. The average price of NADES was estimated to be $2 \mathrm{~S}$ per $\mathrm{kg}$, which is slightly higher than the traditional solvents while obviously lower than ionic liquids sold on the market. ${ }^{49}$ Additionally, NADES are capable of being efficiently recovered and therefore the cost of using NADES could be reduced by reducing solvent consumption. NADES are also environmentally-friendly solvents that produce almost no waste and therefore the cost of handling the waste in production processes could be reduced. However, on the basis of this study, further research is needed if NADES are to be applied in industrial research.

\section{Conclusions}

In the present study, NADES-MAE was applied in extracting ferulic acid from L. chuanxiong for the first time, and an environmentally-friendly and efficient method was established for extracting ferulic acid. The NADES composed of $1: 2(\mathrm{~mol}$ $\mathrm{mol}^{-1}$ ) choline chloride-1,2-propanediol and 30\% water was the solvent with the highest efficiency for extracting ferulic acid, which significantly improved the extraction yield compared to the traditional solvents. The optimal conditions determined by RSM were an extraction time of $20 \mathrm{~min}$, a microwave temperature of $68{ }^{\circ} \mathrm{C}$, and a solvent-to-solid ratio of $30: 1 \mathrm{~mL} \mathrm{~g}^{-1}$. Compared with other extraction methods, higher extraction yields, less time and lower energy consumption were observed while using the NADES-MAE method. In order to explore the application potential, the yields of different methods under the optimal conditions were compared, and the results showed that the heating + stirring method was as effective as MAE, so it can be the alternative to MEA. Additionally, large-scale extraction using the heating + stirring method as the replacement of MAE could be achieved with comparable extraction yields to the small-scale extraction with longer times. It was proven that the optimized conditions using NADES as the extracting solvent has certain potential in industrial application. Moreover, NADES are also safe, green, adjustable and so on. Therefore, NADES are promising green solvents to extract bioactive compounds from natural resources.

\section{Conflicts of interest}

There are no conflicts to declare.

\section{Acknowledgements}

This work was financially supported by the Sichuan Provincial Science and Technology Department Key Program (Grant No. 2018JZ0028).

\section{References}

1 Z. Chen, C. Zhang, F. Gao, Q. Fu, C. Fu, Y. He and J. Zhang, Food Chem. Toxicol., 2018, 119, 309-325.

2 K. J. Chen and K. Chen, Chin. Med. J., 1992, 105, 870-873.

3 J. Ju and J. Du, J. Tradit. Chin. Med., 1995, 15, 183-184.

4 C.-M. Li, Y.-Q. Guo, X.-L. Dong, H. Li, B. Wang, J.-H. Wu, M.-S. Wong and S.-W. Chan, Food Funct., 2014, 5, 2475-2485. 5 R. Yan, S.-L. Li, H.-S. Chung, Y.-K. Tam and G. Lin, J. Pharm. Biomed. Anal., 2005, 37, 87-95.

6 J. Y. Zhan, K. Y. Zheng, K. Y. Zhu, W. L. Zhang, C. W. Bi, J. P. Chen, C. Y. Du, T. T. Dong, D. T. Lau and K. W. Tsim, Planta Med., 2013, 79, 533-537.

7 R. J. Robbins, J. Agric. Food Chem., 2003, 51, 2866-2887.

8 E. Graf, Free Radical Biol. Med., 1992, 13, 435-448.

9 S.-Y. Chung and E. T. Champagne, Food Chem., 2011, 124, 1639-1642.

10 W. Baohua and O.-Y. Jingping, Cardiovasc. Drug Rev., 2005, 23, 161-172.

11 L. Yang, X. Luo, S. Wang and J. Wang, Journal of Yunnan College of Traditional Chinese Medicine, 2007, 30, 14-16.

12 L. Cui and Y. Liu, Qilu Pharmaceutical Affairs, 2005, vol. 9, pp. 560-561.

13 H. Y. Qin, W. Wang and Z. R. Suo, New Technology \& New Products of China, 2009, 18, 19. 
14 S. S. Tong, Heilongjiang Science and Technology Information, 2012, 21, 41.

15 G. Yang, M. Liang, H. Langchong and L. Yunbo, Chinese Traditional Patent Medicine, 1992.

16 G. Huang, F. Ji and W. Yang, Chin. J. Mod. Appl. Pharm., 2007, 3, 26-29.

17 Z. Wei and Q. Tai-qiu, Food Ferment. Ind., 2010, 11.

18 S. Fan, J. Zhang, D. Xiong and C. Zhang, Chin. J. Exp. Tradit. Med. Formulae, 2011, 17, 46-48.

19 H. Lu, X. Tang and B. Li, Hubei Agricultural Sciences, 2016, vol. 55, pp. 2335-2338.

20 J.-L. Liu, S.-L. Zheng, Q.-J. Fan, J.-C. Yuan, S.-M. Yang and F.-L. Kong, Molecules, 2014, 19, 1887-1911.

21 Q. Cui, X. Peng, X.-H. Yao, Z.-F. Wei, M. Luo, W. Wang, C.-J. Zhao, Y.-J. Fu and Y.-G. Zu, Sep. Purif. Technol., 2015, 150, 63-72.

22 I. T. Karabegović, S. S. Stojičević, D. T. Veličković, N. Č. Nikolić and M. L. Lazić, Sep. Purif. Technol., 2013, 120, 429-436.

23 J. He, Z. Shi and W. Chang, J. Liq. Chromatogr. Relat. Technol., 2009, 27, 1769-1784.

24 W. Routray and V. Orsat, Ind. Crops Prod., 2014, 58, 36-45.

25 X. Fang, J. Wang, S. Zhang, Q. Zhao, Z. Zheng and Z. Song, Sep. Purif. Technol., 2012, 86, 149-156.

26 C.-N. Zhao, J.-J. Zhang, Y. Li, X. Meng and H.-B. Li, Molecules, 2018, 23, 2498.

27 C. Yansheng, Z. Zhida, L. Changping, L. Qingshan, Y. Peifang and U. Welz-Biermann, Green Chem., 2011, 13, 666-670.

28 E. L. Smith, A. P. Abbott and K. S. Ryder, Chem. Rev., 2014, 114, 11060-11082.

29 M. de los Ángeles Fernández, J. Boiteux, M. Espino, F. V. Gomez and M. F. Silva, Anal. Chim. Acta, 2018.

30 A. P. Abbott, G. Capper, D. L. Davies, R. K. Rasheed and V. Tambyrajah, Chem. Commun., 2003, 70-71.

31 Y. Dai, G.-J. Witkamp, R. Verpoorte and Y. H. Choi, Anal. Chem., 2013, 85, 6272-6278.
32 Y. Dai, G. J. Witkamp, R. Verpoorte and Y. H. Choi, Food Chem., 2015, 187, 14-19.

33 L. Duan, L.-L. Dou, L. Guo, P. Li and E.-H. Liu, ACS Sustainable Chem. Eng., 2016, 4, 2405-2411.

34 F. J. Gomez, M. Espino, M. A. Fernández and M. F. Silva, ChemistrySelect, 2018, 3, 6122-6125.

35 A. P. Abbott, D. Boothby, G. Capper, D. L. Davies and R. K. Rasheed, J. Am. Chem. Soc., 2004, 126, 9142-9147.

36 M. Ruesgas-Ramón, M. C. Figueroa-Espinoza and E. Durand, J. Agric. Food Chem., 2017, 65, 3591-3601.

37 M. C. Bubalo, N. Ćurko, M. Tomašević, K. K. Ganić and I. R. Redovniković, Food Chem., 2016, 200, 159-166.

38 X. Peng, M. H. Duan, X. H. Yao, Y. H. Zhang, C. J. Zhao, Y. G. Zu and Y. J. Fu, Sep. Purif. Technol., 2016, 157, 249-257.

39 X.-H. Yao, D.-Y. Zhang, M.-H. Duan, Q. Cui, W.-J. Xu, M. Luo, C.-Y. Li, Y.-G. Zu and Y.-J. Fu, Sep. Purif. Technol., 2015, 149, 116-123.

40 Z. Wei, X. Qi, T. Li, M. Luo, W. Wang, Y. Zu and Y. Fu, Sep. Purif. Technol., 2015, 149, 237-244.

41 Z. Wei, Y. Pan, L. Li, Y. Huang, X. Qi, M. Luo, Y. Zu and Y. Fu, J. Sep. Sci., 2014, 37, 3045-3051.

42 Y. Dai, J. van Spronsen, G.-J. Witkamp, R. Verpoorte and Y. H. Choi, Anal. Chim. Acta, 2013, 766, 61-68.

43 Y. Huang, F. Feng, J. Jiang, Y. Qiao, T. Wu, J. Voglmeir and Z.-G. Chen, Food Chem., 2017, 221, 1400-1405.

44 W. Bi, M. Tian and K. H. Row, J. Chromatogr. A, 2013, 1285, 22-30.

45 M. a. C. Gutiérrez, M. a. L. Ferrer, C. R. Mateo and F. del Monte, Langmuir, 2009, 25, 5509-5515.

46 F. Y. Du, X. H. Xiao, X. J. Luo and G. K. Li, Talanta, 2009, 78, 1177-1184.

47 Y. C. Wu, P. Wu, Y. B. Li, T. C. Liu, L. Zhang and Y. H. Zhou, RSC Adv., 2018, 8, 15069-15077.

48 W. LYU, L. Wu, H. Luo, N. Ye, C. Wang, C. Fan and L. Wan, Drug Eval. Res., 2014, 37, 53-57.

49 R. A. Bogaars, Master thesis, Delft University of Technology, 2015. 